See Article page 1558.

\section{Commentary: Much to do about nodules - Improving care with a multidisciplinary pulmonary nodule clinic}

\author{
Melanie P. Subramanian, MD, MPHS
}

With the widespread increasing use of computed tomographic scans and the favorable findings of the National Lung Screening Trial, the number of pulmonary nodules detected either incidentally or through lung cancer screening programs will continue to grow. ${ }^{1}$ In current US practice, patients with pulmonary nodules may be evaluated by a diverse array of providers, including primary care physicians, pulmonologists, and thoracic surgeons. ${ }^{2}$ In addition, computed tomographic scans may be performed and evaluated either by general diagnostic radiologists or by dedicated thoracic radiologists. This can lead to significant heterogeneity in adherence to established surveillance and workup guidelines. A survey of 834 US radiologists revealed that $37 \%$ did not work in an institution that adhered to an established protocol for the management of indeterminate pulmonary nodules. ${ }^{3}$ Multidisciplinary pulmonary nodule clinics have been proposed to provide integrated, evidence-based, and high quality care for the evaluation of patients with pulmonary nodules.

In this issue of the Journal, Madariaga and colleagues ${ }^{4}$ describe their institutional experience with the implementation of a multidisciplinary pulmonary nodule clinic. Specifically, they highlight longitudinal results of patients who were evaluated in the clinic and went on to undergo surgical resection. Their clinic integrated specialists from interventional radiology, medical oncology, radiation oncology, surgery, pulmonology,

\footnotetext{
From the Division of Cardiothoracic Surgery, Washington University School of Medicine, St Louis, Mo.

No funding was provided for this article

Disclosures: Author has nothing to disclose with regard to commercial support.

Received for publication Oct 18, 2019; revisions received Oct 18, 2019; accepted for publication Oct 21, 2019; available ahead of print Nov 25, 2019.

Address for reprints: Melanie P. Subramanian, MD, MPHS, 660 S Euclid Ave, Campus Box 8234, St Louis, MO 63110 (E-mail: m.p.subramanian@email. wustl.edu).

J Thorac Cardiovasc Surg 2020;159:1569-70

$0022-5223 / \$ 36.00$

Copyright (c) 2019 by The American Association for Thoracic Surgery

https://doi.org/10.1016/j.jtcvs.2019.10.108
}

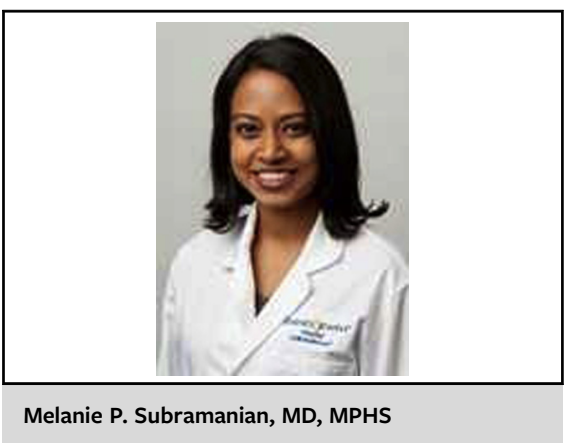

\section{CENTRAL MESSAGE \\ A multidisciplinary nodule clinic can be an effective and efficient strategy to evaluate patients with pulmonary nodules.}

and thoracic radiology. Importantly, they recognized the nuanced differences in risk profile between patients with nodules detected incidentally and patients with nodules detected through lung cancer screening, and they reported their characteristics and treatment courses separately.

Overall, the results were favorable. Madariaga and colleagues ${ }^{4}$ report relatively little use of additional invasive diagnostic tests (ie, computed tomographically or bronchoscopically guided biopsy). In addition, they found that a high percentage of patients with primary lung cancer who underwent surgical resection had the cancer detected at an early stage. Among patients with surgically resected primary lung cancer, the pathologic stage was Ia in $87 \%$ of screening patients and $86.5 \%$ of those with incidental nodule detection. These patients had high overall and disease-free survivals.

The benefits of the multidisciplinary clinic described by Madariaga and colleagues ${ }^{4}$ are clear; the collaboration of experts in multiple disciplines could lead to decreased diagnostic and invasive testing, decreased false-positive results, and earlier time to treatment. Madariaga and colleagues ${ }^{4}$ suggest that their clinic could lead to lower resource utilization; however, an important consideration that must be taken into account is the resources spent on those who did not undergo surgical resection. Madariaga and colleagues ${ }^{4}$ chose not to include longitudinal data for those who did not receive surgical resection. These data, in addition to actual cost data, will be necessary before making final conclusions regarding the cost-effectiveness of such a clinic. In addition, this clinic included a relatively homogeneous 
patient population from a presumably limited geographic distribution. Other institutions that take care of more sociodemographically diverse populations or capture a patient referral base over a significantly wider geographic catchment area may face additional challenges. Nevertheless, the publication of Madariaga and colleagues ${ }^{4}$ demonstrates important and promising findings associated with the use of a multidisciplinary pulmonary nodule clinic. Their clinic serves as an example of an efficient and effective model to care for patients with pulmonary nodules, which will be an ever-growing pool in the future.

\section{References}

1. National Lung Screening Trial Research Team, Aberle DR, Adams AM, Berg CD, Black WC, Clapp JD, et al. Reduced lung-cancer mortality with low-dose computed tomographic screening. N Engl J Med. 2011;365:395-409.

2. Korst RJ. Systematic approach to the management of the newly found nodule on screening computed tomography: role of dedicated pulmonary nodule clinics. Thorac Surg Clin. 2013;23:141-52.

3. Eisenberg RL, Bankier AA, Boiselle PM. Compliance with Fleischner Society guidelines for management of small lung nodules: a survey of 834 radiologists. Radiology. 2010;255:218-24.

4. Madariaga ML, Lennes IT, Best T, Shepard J-AO, Fintelmann FJ, Mathisen DJ, et al; the MGH Pulmonary Nodule Clinic Collaborative. Multidisciplinary selection of pulmonary nodules for surgical resection: diagnostic results and long-term outcomes. J Thorac Cardiovasc Surg. 2020;159:1558-66.e3. 